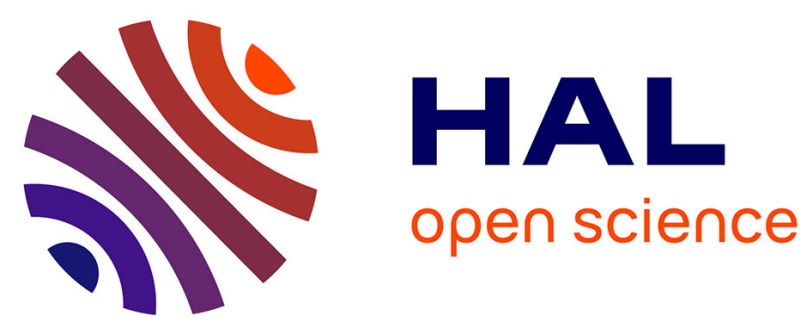

\title{
Dual Inhibition of FLT3 and AXL by Gilteritinib Overcomes Hematopoietic Niche-Driven Resistance Mechanisms in FLT3 -ITD Acute Myeloid Leukemia
}

Pierre-Yves Dumas, Arnaud Villacreces, Amélie Guitart, Ali El-Habhab, Layal Massara, Olivier Mansier, Audrey Bidet, Delphine Martineau, Solene Fernandez, Thibaut Leguay, et al.

\section{To cite this version:}

Pierre-Yves Dumas, Arnaud Villacreces, Amélie Guitart, Ali El-Habhab, Layal Massara, et al.. Dual Inhibition of FLT3 and AXL by Gilteritinib Overcomes Hematopoietic Niche-Driven Resistance Mechanisms in FLT3 -ITD Acute Myeloid Leukemia. Clinical Cancer Research, 2021, 27 (21), pp.6012-6025. 10.1158/1078-0432.CCR-20-3114 . hal-03455205

\section{HAL Id: hal-03455205 \\ https://hal.science/hal-03455205}

Submitted on 30 Nov 2021

HAL is a multi-disciplinary open access archive for the deposit and dissemination of scientific research documents, whether they are published or not. The documents may come from teaching and research institutions in France or abroad, or from public or private research centers.
L'archive ouverte pluridisciplinaire HAL, est destinée au dépôt et à la diffusion de documents scientifiques de niveau recherche, publiés ou non, émanant des établissements d'enseignement et de recherche français ou étrangers, des laboratoires publics ou privés. 
Dual inhibition of FLT3 and AXL by gilteritinib overcomes hematopoietic niche-driven resistance mechanisms in FLT3-ITD acute myeloid leukemia

\section{Authors}

5 Pierre-Yves Dumas ${ }^{1,2}$, Arnaud Villacreces ${ }^{2}$, Amelie Guitart ${ }^{2}$, El-habhab Ali ${ }^{2}$, Layal Massara ${ }^{2}$, Olivier

6 Mansier $^{3,4}$, Audrey Bidet ${ }^{4}$, Delphine Martineau ${ }^{1,2}$, Solene Fernandez ${ }^{2}$, Thibaut Leguay ${ }^{1}$, Arnaud Pigneux ${ }^{1,2}$,

7 Isabelle Vigon², Jean-Max Pasquet ${ }^{2 \#}$, Vanessa Desplat $^{2 \#}$

9 Authors' affiliations

10 1. CHU Bordeaux, Service d'Hématologie Clinique et de Thérapie Cellulaire, F-33000, Bordeaux, France

11 2. Univ. Bordeaux, INSERM, BMGIC, U1035, F-33000 Bordeaux, France

12 3. Institut National de la Santé et de la Recherche Médicale, U1034, 33000, Bordeaux, France

13 4. CHU Bordeaux, Service d'Hématologie Biologique, F-33000, Bordeaux, France

\section{Corresponding authors}

16 Dr Vanessa Desplat, INSERM U1035, 146 rue Léo Saignat, Carreire Bâtiment TP, $4{ }^{\text {ème }}$ étage, Université de 17 Bordeaux, 33076 Bordeaux, France, Tel : 0557571611

18 Mail: vanessa.desplat@u-bordeaux.fr

Dr Pierre-Yves Dumas, INSERM U1035, 146 rue Léo Saignat, Carreire Bâtiment TP, $4{ }^{\text {ème }}$ étage, Université de

21 Bordeaux, 33076 Bordeaux, France, Tel : 0557656511

Mail: pierre-yves.dumas@u-bordeaux.fr

\# Vanessa Desplat and Jean-Max Pasquet are joint last author. 


\section{Word count}

27 Abstract: 199

28 Text: 5183

29 Figures: 6

30 Supplementary tables: 3

31 Supplementary figure: 4

32

33 Running title

34 Gilteritinib and cell-extrinsic mechanisms of AML resistance

35

36 Keywords

37 Acute myeloid leukemia, gilteritinib, quizartinib, FLT3, AXL, microenvironment, hypoxia 38

39 Running title

40 Gilteritinib and cell-extrinsic mechanisms of AML resistance

41

42 Keywords

43 Acute myeloid leukemia, AXL, FLT3, gilteritinib, hypoxia, microenvironment, quizartinib 44 


\section{Abstract}

AXL has been shown to play a pivotal role in the selective response of FLT3-ITD AML cells to FLT3 tyrosine kinase inhibitors (TKI), particularly within the bone marrow microenvironment. Herein, we compared the effect of dual FLT3/AXL TKI Gilteritinib with Quizartinib through in vitro models mimicking hematopoietic niche conditions, ex vivo in primary AML blasts and in vivo with dosing regimens allowing plasma concentration close to those used in clinical trials. We observed that Gilteritinib maintained a stronger proapoptotic effect in hypoxia and coculture with bone marrow stromal cells compared with Quizartinib,

53 linked to a dose-dependent inhibition of AXL phosphorylation. In vivo, use of the MV4-11 cell line with 54 hematopoietic engraftment demonstrated that Gilteritinib was more effective than Quizartinib at targeting 55 leukemic cells in bone marrow. Finally, FLT3-ITD AML patient-derived xenografts revealed that this effect 56 was particularly reproducible in FLT3-ITD AML with high allelic ratio in primary and secondary xenograft.

57 Moreover, Gilteritinib and Quizartinib displayed close toxicity profile on normal murine hematopoiesis, 58 particularly at steady state. Overall, these findings suggest that Gilteritinib as a single agent, compared with 59 Quizartinib, is more likely to reach leukemic cells in their protective microenvironment, particularly AML 60 clones highly dependent on FLT3-ITD signaling.

61 
Acute myeloid leukemia (AML) is a myeloid malignancy carrying a heterogeneous molecular panel of mutations. It participates in a blockade of differentiation and an increased proliferation of myeloid hematopoietic progenitor cells. The Fms-like tyrosine kinase 3 (FLT3) is a class III receptor tyrosine kinase expressed in progenitor cells. Its ligand binds and activates FLT3, inducing a strong activation of PI3K/AKT and MAPK pathways (1). Twenty-five percent of AML patients display internal tandem duplication (ITD) in the juxta-membrane domain of FLT3, leading to constitutive activation of its downstream signaling to which is added the activation of the transcription factor STAT5 (2). This mutation, which carries an adverse prognosis (3), has become an important therapeutic target (4), and its frequent presence at relapse suggests that FLT3-ITD AML-initiating cells are key targets for long-lasting remission.

FLT3 tyrosine kinase inhibitors (FLT3-TKI) have been developed as ATP-competitive inhibitors. Firstgeneration FLT3-TKI have a slight single agent activity but showed clinical efficacy in randomized placebocontrolled trials in association with an intensive chemotherapy $(5,6)$. Second-generation FLT3-TKI, such as Quizartinib and Gilteritinib, are more specific for FLT3 and have recently shown significant activity as a single agent in relapsed/refractory $(R / R) A M L$, with composite complete remissions ranging from $45 \%$ to $55 \%$, associated with prolongation of overall survival (OS) compared with other standards of care $(7,8)$. Quizartinib is a FLT3-TKI that has been specifically optimized pharmacodynamically and pharmacokinetically to inhibit the FLT3-ITD mutant protein. This drug induces clearance of peripheral blasts through induction of apoptosis, whereas cell cycle arrest and terminal myeloid differentiation have been shown in bone marrow blasts (9). Gilteritinib is a dual FLT3 and AXL inhibitor with in vitro activity against both FLT3-ITD and tyrosine kinase domain (TKD) mutations. Gilteritinib allows sustained FLT3 inhibition; response rates are close to those observed with quizartinib, but complete remissions are much more frequent. Gilteritinib induces two distinct bone marrow responses in FLT3-mutated AML, with and without differentiation of leukemic cells (10). 
89 chemotaxis, apoptotic body clearance and immunity in physiological conditions (11). Moreover, AXL is over-

90 expressed in a broad range of cancers and is regularly associated with a poor prognosis. In AML, AXL and

91 GAS6 levels of expression have been related to poor outcomes where paracrine AXL activation has been

92 shown to induce $A M L$ resistance to various therapies $(12,13)$. Finally, we previously reported that several

93 microenvironment-related mechanisms, such as hypoxia and STAT5-activating cytokines, converge to

94 enhance AXL expression and activation, thereby sustaining pro-survival signals that selectively protect FLT3-

95 ITD AML cells in the hematopoietic niche (14).

96 Here, we were interested in testing the efficacy of Gilteritinib, as a dual FLT3 and AXL inhibitor, in AML cells

97 within their specific microenvironment. We show that Gilteritinib retains efficacy in vitro in FLT3-ITD AML

98 despite bone marrow stromal cells (BMSC) coculture and hypoxia compared with Quizartinib. Moreover, ex

99 vivo primary AML blasts display same profile of response and in various AML mouse models, we show that

100 Gilteritinib is particularly relevant in FLT3-ITD AML with high allelic ratio. Finally, we also studied toxicity

101 profile of Gilteritinib and Quizartinib on normal murine hematopoiesis in two mice strains.

102 


\section{Cell culture}

MV4-11, MOLM-13 and MOLM-14 are 3 AML cell lines. MV4-11 has a 48, XY, t(4;11)(q21;q23), +8, +19 karyotype with a $30 \mathrm{bp} \mathrm{FLT3-ITD} \mathrm{mutation.} \mathrm{MOLM-13} \mathrm{has} \mathrm{the} \mathrm{following} \mathrm{karyotype} 51(48-52)<2 \mathrm{n}>\mathrm{XY},+8,+8$, $+8,+13$, del(8)(p1?p2?), ins(11;9)(q23;p22p23) carrying KMT2A-MLLT3 mutations and a 21 bp FLT3-ITD mutation; finally, MOLM-14 has the following karyotype $-49(46-50)<2 n>X Y,+6,+8,+13$, $\operatorname{der}(2) t(1 ; 2)(q 31 ; q 35)$, ins(11;9)(q23;p22p23), del(14(q23q32.3), del(16)(q11.2q13.1) including also KMT2A

111 rearrangement and a $21 \mathrm{bp}$ FLT3-ITD mutation. All AML and bone marrow stromal cell lines (MV4-11, 112 MOLM-13, MOLM-14, MS5) were cultured in aMEM medium, supplemented with $10 \%$ fetal calf serum 113 (FCS), $2 \mathrm{mM}$ L-glutamine, $50 \mathrm{U} / \mathrm{mL}$ penicillin, and $50 \mu \mathrm{g} / \mathrm{mL}$ streptomycin. Hypoxia at $1 \%$ was induced by 114 incubating cells in a specific oxygen $\left(\mathrm{O}_{2}\right)$ chamber (BioSpherix). Stromal cells were implanted for 3 days to 115 reach $80 \%$ confluence, then culture medium was removed, and leukemic cells were added to the flask. Co116 culture cells were incubated either in a specific $\mathrm{O}_{2}$ chamber for $1 \% \mathrm{O}_{2}$ hypoxic culture or in an incubator at $1175 \% \mathrm{CO}_{2}$ for normoxic culture. Cells were cultured with vehicle or gilteritinib (ASP2215; kindly provided by 118 Astellas Pharma) or quizartinib (AC220; LC Laboratories), both dissolved in dimethyl sulfoxide (DMSO).

\section{Cell proliferation assay}

121 Cell proliferation assays were performed using MTS tetrazolium (Cell Titer96 Aqueous; Promega). Cells $\left(10^{5}\right)$ were plated and incubated in quadruplicate into microtiter-plate wells in $100 \mu \mathrm{L}$ of culture medium with various doses of gilteritinib. For the analyses, plates were incubated for $3 \mathrm{~h}$ with $20 \mu \mathrm{L}$ of MTS and read in a microplate auto reader (Imark Biorad) at $490 \mathrm{~nm}$. Results are expressed as the mean optical density of the four-well set for each gilteritinib dose. All experiments were repeated at least three times during the

\section{Western blot analysis}


Protein lysates were prepared with cell lysis buffer 10x (Cell Signaling Technology) and protein

130 concentrations were determined by $\mathrm{BCA}^{\mathrm{TM}}$ protein assay (Pierce). Equal amounts of proteins were resolved 131 on $12 \%$ polyacrylamide gel and transferred to a nitrocellulose or PVDF membrane (TurboBlot; Biorad). After 132 membrane saturation, the blots were incubated with the respective antibody followed by an anti-rabbit or 133 anti-mouse peroxidase-conjugated secondary IgG antibody (Table S1) and revealed with an enhanced 134 chemiluminescence detection method (Western Lightning Chemiluminescence Reagent Plus; Perkin Elmer). 135 Quantification of protein expression was performed by densitometry using Image Lab software (Biorad). 136 Phosphorylation of protein expression was normalized using total protein expression by calculating the 137 ratio of the phosphorylated protein level to their total protein level counterparts. The ratio of the control condition was normalized to 1 .

\section{Flow cytometric analysis}

141 Cell apoptosis was assessed using an APC-conjugated Annexin V labeling detection kit coupled to flow 142 cytometry and BD FACSDIVA ${ }^{\mathrm{TM}}$ software (BD Bioscience). For normal hematopoiesis analyses, BM cells were 143 obtained by crushing tibias, femurs and hip with a pestle and mortar. Filtered single cell suspensions were 144 incubated in Fc block and then stained with antibodies. For NSG analyses, following incubation with Fc 145 block, unfractionated BM cell suspensions were stained with lineage markers containing biotin-conjugated 146 anti-CD4, anti-CD5, anti-CD8a, anti-CD11b, anti-B220, anti-Gr-1 and anti-Ter119 antibodies together with 147 APC-conjugated anti-c-Kit. When applicable, to distinguish hCD45 AML xenotransplant cells from mCD45 148 recipient mice cells, FITC-conjugated anti-hCD45 and APC-Cy7-conjugated anti-mCD45 antibodies were 149 included in the antibody cocktail described above. For C57BL/6J analysis, following incubation with Fc 150 block, unfractionated BM cell suspensions were stained with lineage markers containing biotin-conjugated 151 anti-CD4, anti-CD5, anti-CD8a, anti-CD11b, anti-B220, anti-Gr-1 and anti-Ter119 antibodies together with 152 APC-conjugated anti-c-Kit, BV421-conjugated anti-Sca-1, AF700-conjugated anti-CD48, PE-Cy7-conjugated 153 anti-CD150, FITC-conjugated anti-CD34 and PE-conjugated anti-CD135. Antibodies clones are detailed Table 154 S2. Flow cytometry analyses were performed using a LSR Fortessa (BD). Analysis was done using FlowJo v.X 155 (BD). 


\section{Animal models for in vivo studies with subcutaneous xenografts and bioluminescent imaging}

158 NOD.Cg-Prkdc ${ }^{\text {scid }}|| 2 \mathrm{rg}^{\mathrm{tm} 1 \mathrm{Wjl}_{\mathrm{j}}} / \mathrm{SzJ}$ immunodeficient mice (NSG) were bred at the University of Bordeaux's

159 animal facility for experiments validated by the French ministry (authorization no. 00048.2). Female

160 animals were included in protocols at 8 weeks old and were monitored weekly for body weight. For

161 subcutaneous xenografts, prior to implantation, cells were resuspended in 25\% Matrigel (BD Biosciences)

162 and 75\% culture medium without FCS. Female NSG mice received injections into their hind flanks with 1.75

$163 \times 10^{6}$ cells. Tumor volume was determined three times per week with a caliper and calculated by the

164 formula $\left(I^{2 *} \mathrm{~L}\right) / 2$. When tumors reached $400-500 \mathrm{~mm}^{3}$, treatment started with quizartinib $(5 \mathrm{mg} / \mathrm{kg} / \mathrm{day}$

165 body weight) or gilteritinib $(5,10$, and $30 \mathrm{mg} / \mathrm{kg} /$ day body weight) by daily oral gavage. For MV4-11

166 hematopoietic engraftment, NSG mice were conditioned with intraperitoneal injections of busulfan (Pierre

167 Fabre) at $20 \mathrm{mg} / \mathrm{kg} /$ day for 2 days, and then injected intravenously with $10^{6} \mathrm{MV} 4-11$ luciferase-transduced

168 cells $\left(10^{6}\right.$ cells $\left./ 100 \mu \mathrm{L}\right)$ at day 0 . At day 7 , engraftment was analyzed by whole bioluminescent imaging (BLI)

169 before treating mice with vehicle or quizartinib $(5 \mathrm{mg} / \mathrm{kg} /$ day body weight) or gilteritinib $(30 \mathrm{mg} / \mathrm{kg} / \mathrm{day}$

170 body weight) by daily oral gavage for 7 days. At day 14 and day 20, mice were injected intraperitoneally

171 with firefly luciferase substrate D-luciferin $(150 \mathrm{mg} / \mathrm{kg})$ before imaging using a photon bioimager and 172 M3Vision software (Biospace Lab).

\section{Primary AML patient samples and patient-derived xenografts cells generation}

175 Patients at the University Hospital of Bordeaux gave written informed consent for the use of biological samples for research, in accordance with the Declaration of Helsinki. AML cells were from a 57-year-old woman diagnosed with a de novo AML 4 in FAB classification with normal karyotype FLT3-ITD with an ITD/wild type ratio at 0.82 at diagnosis and a NPM1 mutation. Flow cytometry profile was CD34, HLA-DR-, $\mathrm{CD}^{\circ}, \mathrm{CD}^{-} 3^{+}, \mathrm{CD}^{+} 3^{+}, \mathrm{CD} 117^{+}, \mathrm{MPO}^{+}, \mathrm{CD}^{-}$and $\mathrm{CD}^{-}$. Patient-derived xenografts (PDX) were generated by 180 retro-orbital injections of $10^{6}$ viable primary AML cells in 8-week-old female NSG mice, previously 181 conditioned over 2 days by intraperitoneal injections of busulfan (Pierre Fabre; $20 \mathrm{mg} / \mathrm{kg} /$ injection/day). 182 Bone marrow engraftment of primary human AML cells in each mouse was followed monthly by 
intrafemoral aspiration and flow cytometry analyses. When levels reached at least $10 \%$ human AML cells

184 (CD33 ${ }^{+} \mathrm{CD}_{4} 5^{+}$; see Table S2 for the antibodies used), the mice were sacrificed and bone marrow cells were 185 collected, pooled, and cryopreserved (Cryostor CS10; Stem Cell Technologies). The FLT3 status of human 186 AML cells (hereafter AML PDX cells) in bone marrow was assessed as described previously (15). Two 187 batches of AML PDX cells were generated. The first batch was obtained from two mice sacrificed 6 weeks 188 post-transplantation with an ITD length at $45 \mathrm{bp}$ and an FLT3-ITD/wild-type ratio of 0.44 and FLT3-ITD/FLT3 189 total ratio of 0.30 , which demonstrated the presence of FLT3-ITD and wild-type AML clones. The second 190 batch was obtained from five mice sacrificed 12 weeks post-transplantation. All human cells in this second 191 batch carried just a FLT3-ITD of 45 bp, which demonstrated a loss of heterozygosity (LOH).

\section{Animal models for in vivo studies from AML PDX cells transplantation}

AML PDX cells were injected by retro-orbital route in nonconditioned 8-week-old female NSG mice $\left(10^{6}\right.$ viable PDX cells/mouse). After AML PDX engraftment ( $>1 \%$ of human cells in bone marrow), 4-5 mice/group were treated with vehicle (10\% DMSO in water) or quizartinib (5 mg/kg/day body weight) or gilteritinib ( $30 \mathrm{mg} / \mathrm{kg} /$ day body weight) by daily oral gavage for 2 weeks. Mice were sacrificed three days after the last gavage and BM collected from the whole rear limb (hip, femur and tibia). AML PDX cells and murine normal hematopoiesis were analyzed by flow cytometry as described previously (16).

\section{Animal models for in vivo studies in normal murine hematopoiesis}

For both NSG and $\mathrm{C} 57 \mathrm{BI} / \mathrm{J}$ model, 5 mice/group were treated with vehicle (10\% DMSO in water) or quizartinib ( $5 \mathrm{mg} / \mathrm{kg} /$ day body weight) or gilteritinib ( $30 \mathrm{mg} / \mathrm{kg} /$ day body weight) by daily oral gavage for 2 weeks. Animals were either sacrificed for BM analysis 3 days post-treatment or kept alive to assess complete blood count over time for a month. Complete Blood count were measured using a Scil Vet ABC Plus (Horiba). Colony forming cells (CFC) assays : CFC assays were performed using MethoCultTM M3434 day 12. 
210 Animal models for in vivo studies from AML PDX cells transplantation in secondary recipient

211 Live AML PDX cells (10 $0^{6}$ DAPI-negative cells) of each mouse recipient were collected and transplanted for

212 secondary xenografts in nonconditioned 8-week-old female NSG mice. At 9-10 weeks post-transplantation,

213 the mice were sacrificed and AML PDX cells and murine normal hematopoiesis were analyzed by flow 214 cytometry as described previously (16).

216 Statistical analyses

217 All analyses were performed using GraphPad Prism software. Unless otherwise indicated, data are 218 presented as the mean \pm standard error of the mean. Statistical significance was calculated using the 219 Student's t-test unless otherwise stated. Differences with $\mathrm{p}$ values $<0.05$ were considered statistically 220 significant with *, $\mathrm{p}<0.05 ;{ }^{* *}, \mathrm{p}<0.01 ;{ }^{* *}, \mathrm{p}<0.001 ; \mathrm{NS}$, nonsignificant. 
Gilteritinib exerts antileukemic effects and inhibits AXL despite stromal cells coculture

We first validated the inhibitory effect of increasing doses of gilteritinib on three human FLT3-ITD

AML cell lines also expressing AXL. The drug inhibited proliferation of the three cell lines in a dose-

dependent manner with $\mathrm{IC}_{50}$ values at $3.3 \pm 0.6 \mathrm{nM}$ for MV4-11, $19.0 \pm 3.2 \mathrm{nM}$ for MOLM-13, and $25.0 \pm 1.0$

$\mathrm{nM}$ for MOLM-14 (Figure 1A). Additionally, gilteritinib induced a pro-apoptotic effect, as shown by an increased frequency of annexin $\mathrm{V}^{+}$cells: $38.9 \pm 0.8 \%$ for MV4-11, $54.9 \pm 2.0 \%$ for MOLM-13, and $90.2 \pm 0.8 \%$ for MOLM-14 at $90 \mathrm{nM}$ for $48 \mathrm{~h}$ (Figure 1B). Similar results were obtained at $72 \mathrm{~h}$ in all conditions tested (data not shown). These anti-proliferative and pro-apoptotic effects were correlated with decreased $\mathrm{Y}_{591} \mathrm{FLT3}$ and $\mathrm{Y}_{779} \mathrm{AXL}$ phosphorylation as well as decreased activation of its downstream signaling. Indeed, densitometry showed dose-dependent decrease in $\mathrm{Y}_{779} \mathrm{AXL}$ phosphorylation in three AML cell lines (1 vs $0.3 ; 0.1 ; 0.2 ; 0.0$ (MV4-11); 1 vs $1 ; 0.7 ; 0.3 ; 0.2$ (MOLM-13); and 1 vs $0.5 ; 0.3 ; 0.2 ; 0.1$ (MOLM-14), for treatment with Gilteritinib at $5 \mathrm{nM}, 20 \mathrm{nM}, 50 \mathrm{nM}$ and $100 \mathrm{nM}$, respectively, compared to vehicle treatment. The decrease in FLT3 phosphorylation was also confirmed (1 versus $0.7 ; 0.5 ; 0.6 ; 0.4$ (MV4-11); 1 versus $0.7 ; 0.4 ; 0.6 ; 0.4$ (MOLM-13); and 1 versus $0.6 ; 0.4 ; 0.4 ; 0.3$ (MOLM-14), for treatment with 238 Gilteritinib at $5 \mathrm{nM}, 20 \mathrm{nM}, 50 \mathrm{nM}$ and $100 \mathrm{nM}$, respectively, compared to vehicle treatment. Finally, data 239 quantifications in figure 1C, also showed a dose-dependent decrease in phosphorylation levels for $\mathrm{pY}_{694} \mathrm{STAT5}, \mathrm{pSer}{ }_{473} \mathrm{AKT}$, and $\mathrm{pT}_{202} / \mathrm{Y}_{204} \mathrm{ERK}$ for all 3 cell lines.

\section{Antileukemic effects of gilteritinib in hypoxia and with stromal cells coculture compared with quizartinib}

The role of AXL in resistance to FLT3 inhibition has been demonstrated previously $(13,14)$. We were

FLT3 inhibition by a highly selective drug such as Quizartinib, particularly in the context of the 
marrow environment. In the absence of BMSC, Gilteritinib and Quizartinib induced quite similar apoptosis in the three FLT3-ITD AML cell lines in normoxia or in hypoxia at $1 \% \mathrm{O}_{2}$ (Figure $2 \mathrm{~A}$ left panel). We previously demonstrated that without BMSC, the level of AXL expression in AML and the level of autocrine secretion of its ligand GAS6 are very low. In contrast, and as demonstrated in a previous work (14), we observed an upregulation of AXL in hypoxia and in BMSC co-culture condition (Figure 2B). Finally, we observed a significant increase in apoptosis induced by Gilteritinib in hypoxia with BMSC compared with normoxia: $15.8 \pm 1.7 \%$ (MOLM-14), respectively (Figure $2 \mathrm{~A}$ right panel).

Gilteritinib compared with Quizartinib, particularly in culture conditions associating hypoxia and stromal cells (Figure 2B). However, this decrease of $\mathrm{Y}_{779} \mathrm{AXL}$ phosphorylation was not correlated with a strong inhibition of ERK and AKT which remained similar for Quizartinib and Gilteritinib suggesting that MAPK and PI3K pathways were mainly dependent of FLT3 signaling (Figure 2B). Next, we investigated whether such effects were observed ex vivo in primary AML blasts. Results are shown in Figure $2 \mathrm{C}$ and patients are described in Table S3. These data showed that Gilteritinib $100 \mathrm{nM}$ allows a trend $(p=0.06)$ to induce a higher apoptotic response in AML blast in co-culture as compared to Quizartinib $3 \mathrm{nM}$. Taken together, these results indicate that Gilteritinib is more efficient in conditions mimicking the hematopoietic niche, suggesting that it could overcome protective mechanisms mediated by the bone marrow microenvironment in vivo.

\section{Antileukemic effects of gilteritinib compared with quizartinib in mouse models xenografted with MV4-11}

Encouraged by the in vitro data suggesting a therapeutic benefit of dual FLT3/AXL inhibition in the 
vitro, we determined dosing regimens in mice for Quizartinib and Gilteritinib that induced close levels of response in a subcutaneous model of FLT3-ITD AML cell line. We subcutaneously xenografted MV4-11 cells into immunodeficient NSG mice. When the tumor size reached $400-500 \mathrm{~mm}^{3}$, the mice were treated by Gilteritinib five times per week for 2 weeks with $5 \mathrm{mg} / \mathrm{kg} /$ day, $10 \mathrm{mg} / \mathrm{kg} /$ day, and $30 \mathrm{mg} / \mathrm{kg} / \mathrm{day}$, or 5 $\mathrm{mg} / \mathrm{kg} /$ day of Quizartinib, which was previously determined to be an effective dose in vivo (14). According to guidelines for dose conversion between animals and humans (17) and the quantitative determination of quizartinib in mouse plasma (18), we expected that $5 \mathrm{mg} / \mathrm{kg} /$ day corresponds to a dosing regimen ranging from $40-50 \mathrm{mg} /$ day in humans $(19,20)$. At day 28 , the median tumor volume was increased to $777.1 \mathrm{~mm}^{3}$ with $5 \mathrm{mg} / \mathrm{kg} /$ day of gilteritinib, whereas it decreased to $84.2 \mathrm{~mm}^{3}$ with $10 \mathrm{mg} / \mathrm{kg} /$ day of gilteritinib. Mice were in complete remission at day 28 with Quizartinib $5 \mathrm{mg} / \mathrm{kg} /$ day and Gilteritinib $30 \mathrm{mg} / \mathrm{kg} /$ day with the same response profile, and at day 40 with Gilteritinib $10 \mathrm{mg} / \mathrm{kg} /$ day. Treatment by FLT3-TKI was discontinued when mice were in complete remission, except for mice in the Gilteritinib $5 \mathrm{mg} / \mathrm{kg} / \mathrm{day}$ arm, which were sacrificed at day 28 for ethical reasons (Figure 3A). Finally, median time to relapse was 13 days for Quizartinib $5 \mathrm{mg} / \mathrm{kg} /$ day arm and 14 days for Gilteritinib $30 \mathrm{mg} / \mathrm{kg} /$ day arm ( $p=\mathrm{NS}$ ) whereas it was at 2 days for Gilteritinib $10 \mathrm{mg} / \mathrm{kg} /$ day arm ( $<<0.001$ compared with the two other arms) (Figure 3B). Based on the quantitative determination of the gilteritinib in mouse plasma (21), we expected that $30 \mathrm{mg} / \mathrm{kg} / \mathrm{day}$ corresponds to a dosing regimen at 120 to $130 \mathrm{mg} /$ day in humans (22). Finally, both dosing regimens coincided with clinically relevant posology used as single agents in clinical trials.

This strategy allowed us to compare the specific effect of such doses considering engraftment in the bone marrow environment in the next experiment. We first used FLT3-ITD MV4-11 cells that were xenografted by vein injection. Leukemic cell engraftment was analyzed by bioluminescence imaging (BLI) as described previously (14). At day 7, one group of the mouse cohort was treated by daily oral gavage for 14 days with Quizartinib ( $5 \mathrm{mg} / \mathrm{kg} /$ day), one group by Gilteritinib ( $30 \mathrm{mg} / \mathrm{kg} /$ day), and a control group with vehicle. Response to treatment was assessed by BLI at day 14, and then at day 20 (Figure 3C). At day 14, a significant decrease in total bioluminescence signal was observed in response to Quizartinib compared with 301 the control group $\left(4.3 \times 10^{7} \mathrm{Ph} / \mathrm{s} / \mathrm{sr}\right.$ versus $\left.1.5 \times 10^{6} \mathrm{Ph} / \mathrm{s} / \mathrm{sr}, \mathrm{p}<0.001\right)$ and in response to Gilteritinib 302 compared with the control group $\left(4.3 \times 10^{7} \mathrm{Ph} / \mathrm{s} / \mathrm{sr}\right.$ versus $\left.0.8 \times 10^{6} \mathrm{Ph} / \mathrm{s} / \mathrm{sr}, \mathrm{p}<0.001\right)$. This leukemic 
burden decrease was stronger at day $14\left(0.8 \times 10^{6} \mathrm{Ph} / \mathrm{s} / \mathrm{sr}\right.$ versus $\left.1.5 \times 10^{6} \mathrm{Ph} / \mathrm{s} / \mathrm{sr}, \mathrm{p}<0.05\right)$ and at day 20

$304\left(0.5 \times 10^{6} \mathrm{Ph} / \mathrm{s} / \mathrm{sr}\right.$ versus $\left.1.3 \times 10^{6} \mathrm{Ph} / \mathrm{s} / \mathrm{sr}, \mathrm{p}<0.05\right)$ with Gilteritinib compared with Quizartinib, 305 respectively (Figure 3C).

Antileukemic effects of Gilteritinib and Quizartinib in FLT3-ITD AML patient-derived xenografts Encouraged by these in vivo results with the FLT3-ITD AML cell line MV4-11, we explored the effects of these two FLT3-TKI in vivo on two different batches of AML-PDX cells generated from a FLT3-ITD AML patient using the process presented in Figure 4A. The first batch contained FLT3-ITD and wild-type AML 311 cells with an ITD/wild-type ratio of 0.44 . The second batch contained FLT3-ITD AML cells with LOH. We 312 transplanted AML PDX cells of these two batches into NSG mice and monitored engraftment in bone 313 marrow using flow cytometry (CD45/CD33 double staining). Then, mice were treated by daily oral gavage 314 with Quizartinib (5 mg/kg/day) and Gilteritinib (30 mg/kg/day) for 2 weeks. Response to treatment was 315 assessed by flow cytometric analysis of human $\mathrm{CD} 45^{+} / \mathrm{CD} 33^{+}$blasts from crushed femurs, tibias, and hips 316 (Figure 4B). Analysis of the results based on molecular characterization of the transplanted batches 317 confirmed that mean percentage of human $\mathrm{CD}_{4} 5^{+} / \mathrm{CD} 3^{+}$blast cells in the bone marrow at week 9 post318 transplantation was significantly higher in Quizartinib-treated mice $(27.8 \% \pm 5.8)$ than in Gilteritinib-treated 319 mice $(15.4 \% \pm 2.5)$ in FLT3-ITD AML PDX with LOH (Figure 4C). Conversely, mean percentage of human $320 \mathrm{CD} 5^{+} / \mathrm{CD}_{3} 3^{+}$blast cells in the bone marrow at week 9 post-transplantation was similar in Quizartinib321 treated mice and in Gilteritinib-treated mice in FLT3-ITD AML PDX with an ITD/wild-type ratio of 0.44 322 (Figure 4D). These results suggested that gilteritinib displayed a higher level of efficacy in BM microenvironment for AML with high FLT3-ITD/wt ratio.

Gilteritinib spared normal murine hematopoiesis in a BM microenvironnement impaired by AML signaling. Three days post-FLT3-TKI treatment, there were no differences in BM cellularity between vehicle- 
330 (Figure 5A, left panel). In contrast, in AML-PDX NSG group, murine leukocytes cellularity was significantly 331 higher in gilteritinib-treated mice (median $89.3 \times 10^{6}$, IQR 83.7-95.3) than in quizartinib-treated mice 332 (median $73.0 \times 10^{6}$, IQR 45.1-78.6) (Figure 5A, right panel). Finally, comparing across transplanted and non333 transplanted groups, we showed that murine BM cellularity was returned to normal only with Gilteritinib. 334 When separating murine leukocytes between differentiated precursor cells defined as $\mathrm{mCD}^{+} 5^{+} \mathrm{Lin}^{+}$cells 335 (Figure $5 \mathrm{~B}$ ) and a broader immature population defined as $\mathrm{mCD}^{+} 5^{+}$Lin $^{-}$cells (Figure $5 \mathrm{C}$ ), the same 336 conclusions can be drawn. We next decided to investigate the more primitive compartments (Figure S3A) 337 such as HSPC using mCD45 ${ }^{+}$Lin $^{-} \mathrm{c}^{-K i t^{H i g h}}$ immunophenotyping approach: there were here some differences 338 between vehicle-treated mice, quizartinib-treated mice and gilteritinib-treated mice in the non339 transplanted NSG group suggesting a slight impairment of this compartment induced by FLT3-TKI, 340 particularly Gilteritinib (median $5.7 \times 10^{6}$ [IQR $5.3-6.3$ ] vs $4.6 \times 10^{6}$ [IQR 4.4-5.3] vs $4.2 \times 10^{6}$ [IQR $3.9-4.4$ ] in 341 Vehicle, Quizartinib and Gilteritinib, in non-transplanted NSG, respectively). However, the absolute 342 numbers of murine $\mathrm{mCD}^{4} 5^{+} \mathrm{Lin}^{-} \mathrm{C}-\mathrm{Kit}^{\mathrm{High}}$ cells were similar in both Gilteritinib-treated mice (median $3.5 \times 10^{6}$

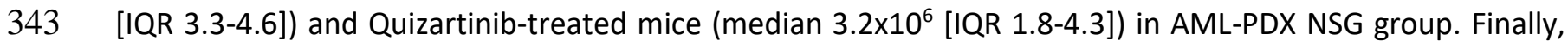
344 as observed for mature compartments, Gilteritinib was able to maintain the level of $\mathrm{mCD} \mathrm{C5}^{+} \mathrm{Lin}^{-} \mathrm{c}-\mathrm{Kit}^{\mathrm{High}}$ 345 cells in AML-PDX NSG group close to the one observed in Gilteritinib-treated non-transplanted NSG group 346 whereas Quizartinib did not (Figure 5D). It should nevertheless be noted that outside the leukemic context, 347 Gilteritinib impairs normal primitive murine hematopoiesis more than Quizartinib (Figure 5C and 5D, left 348 panels). Taken together, these data could suggest either a better efficacy of Gilteritinib in BM clearance of 349 AML blasts, leading to a better murine hematopoiesis, or a toxicity of Quizartinib on normal murine 350 hematopoiesis specifically in the context of AML, since this point is not observed in non-transplanted NSG 351 mice. To discriminate between these two hypotheses, we analyzed murine hematopoiesis according to BM 352 clearance as previously shown in Figure 4C and 4D. In " $\mathrm{LOH}$ " group, BM clearance was higher with 353 Gilteritinib, whereas in "ratio 0.44", BM clearance was the same with both treatments. In both FLT3-ITD354 AML-PDX group "LOH" and "ratio 0.44", independently of AML blasts clearance, murine leukocyte numbers 355 were higher upon Gilteritinib treatment compared with Quizartinib treatment (Figure 5E). This rules in 
favour of a lower toxicity for Gilteritinib for maintaining normal murine hematopoiesis in a BM microenvironnement impaired by AML.

In front of these results, we next assessed HSPC functions. First, we performed CFC assay at sacrifice of non-transplanted NSG mice and found that none of the FLT3-TKI impaired progenitors' ability to generate colonies (Figure 5F). Then, we analyzed Complete Blood Count (CBC) at day 4, day 18 and day 31 post-TKI treatment in a non-transplanted mice group. We did not find any clinically relevant effects of either TKI, Quizartinib or Gilteritinib, supporting the fact that these treatments are well tolerated at theses doses on a steady-state hematopoiesis whether on WBC, Red blood cells, platelets, lymphocytes, monocytes, or granulocytes (Figure S1). To confirm our conclusions, we performed a new set of experiments on $\mathrm{C} 57 \mathrm{BI} / 6 \mathrm{~J}$ mice. During treatment, complete blood count was followed and did not show any differences between the 3 groups (Figure S2). As observed in NSG counterparts, three days post-FLT3TKI treatment, there were no significant differences between vehicle-treated mice, quizartinib-treated mice and gilteritinib-treated mice, for the following cell subsets : Lin ${ }^{-}$cells (Figure 5G), Lin $^{-}$Sca1 $^{+}{ }^{-}$-Kit $^{\text {High }}$ cells (Figure $5 \mathrm{H}$ ) and $\mathrm{HSC}$ immunophenotypically defined as LSK CD48- CD150+ $\mathrm{CD} 135^{-} \mathrm{CD}^{-} 4^{-}$(Figure 5I/S3B). We also analyzed CFU-GEMM, -GM, -G, -M and Red/Mk (Figure 5J) without noticing any significant differences. Taken together, these data support the fact that Gilteritinib and Quizartinib display close toxicity profile on normal murine hematopoiesis at steady state, even if Gilteritinib display a slight impairment of primitive compartment without impact on CBC. Conversely, Quizartinib display higher toxicity on murine

Effects of gilteritinib and quizartinib on leukemic initiating cell compartment in secondary AML PDX recipient

To further explore the ability of both FLT3-TKI to target leukemic initiating cells in their microenvironment, we performed secondary transplants from bone marrow of Gilteritinib-treated and Quizartinib-treated AML PDX cells ( $10^{6}$ AML PDX alive cells/secondary recipient) as described in Figure 4B. 381 Once again, analysis of the results (Figure S4) based on molecular characterization of the engrafted batch 382 revealed that gilteritinib was more effective than quizartinib in controlling leukemic stem cell (LSC) 
383 engraftment and leukemic burden in FLT3-ITD AML PDX with LOH (Figure 6A) compared with FLT3-ITD AML

384 PDX with an ITD/wild-type ratio at 0.44 (Figure 6B), in which Quizartinib and Gilteritinib displayed the same 385 levels of efficacy.

386 


\section{Discussion}

Here, we demonstrated that dual AXL and FLT3 inhibition should target FLT3-ITD leukemic cells protected from cytotoxic treatments by various mechanisms including low $\mathrm{O}_{2}$ concentration, BMSC, and soluble factors in the bone marrow microenvironment. AXL is ectopically- or over-expressed in a wide variety of cancers and has always been associated with a poor prognosis (23). We have reported resistance mechanisms involving $A X L$ in chronic myeloid leukemia (24). In AML, AXL and GAS6 levels of expression have been related to poor outcomes $(25,26)$. Paracrine $A X L$ activation has been shown to induce AML resistance to conventional chemotherapies but also to FLT3-targeted therapy $(12,13,27,28)$. We previously demonstrated that AXL was particularly relevant in the setting of the hematopoietic niche (14), suggesting that Gilteritinib could be a candidate to improve FLT3-ITD AML-initiating cell eradication.

We confirmed that Gilteritinib induced dose-dependent antiproliferative and pro-apoptotic effects associated with an inhibition of FLT3 activation and its downstream signaling (21). We demonstrated the specific interest of Gilteritinib in the setting of hypoxia and BMSC coculture, linked to a better efficacy compared with Quizartinib, another FLT3-TKI much more specific of FLT3. Gilteritinib also provided better response to treatment in bioluminescent imaging with a mouse model xenografted with a FLT3-ITD AML cell line. Then, in a FLT3-ITD AML PDX model, we found that Gilteritinib as single agent was more effective in controlling FLT3-ITD AML with a high allelic ratio, whereas its efficacy was similar to that of Quizartinib for AML with FLT3-ITD and FLT3 wild-type clones. Finally, by performing a secondary xenograft, we reproduced identical results but neither Quizartinib nor Gilteritinib fully eradicated leukemic initiating cells. Nevertheless, Gilteritinib-treated AML PDX displayed extended time in terms of leukemic burden, specifically in the FLT3-ITD AML with LOH batch. We could speculate that such efficacy by continuous treatment longer than 2 weeks could perhaps enable deeper impairment of FLT3-ITD AML clone. Finally, we could also speculate that other kinases inhibited by Gilteritinib could also play a role in the bone marrow microenvironment such as LTK or ALK (21). Indeed, effects of mesenchymal stromal cells and endothelial cells on AML cells have been investigated through contact factors and cytokine-mediated crosstalk. Several soluble mediators are known to be linked with remodeling of the HSC niche into a leukemic-permissive 
414

415

niche such as angiogenic growth factors like VEGF, Ang-1, pro-inflammatory cytokines such as IL-6, homingdisrupting cytokines such as CXCL12. Other studies also detected variation according to osteoblastic or adipocytic MSC differentiation, but also immune cells secreting FLT3-ligand or IL-1 (29).

Using two FLT3-ITD AML PDX models, we showed that quizartinib was as effective as gilteritinib when PDX displayed both FLT3-ITD and wild-type clones. These experiments reproduced outcomes of phase 2 clinical trials in which FLT3-TKI as single agent allowed $12 \%$ and $45 \%$ of overall response in FLT3 wild-type AML for gilteritinib (30) and quizartinib (31), respectively. Quizartinib is known to inhibit KIT phosphorylation, leading to an impaired hematopoiesis (7). If Gilteritinib does not inhibit KIT, its use in routine clinical practice unveils also a certain impairment of normal hematopoiesis, independently of its ability to induce blast differenciation $(9,10)$. Here, we provide some data regarding murine hematopoiesis, demonstrating that Gilteritinib and Quizartinib display close toxicity profile on normal murine hematopoiesis at steady state without impact on $\mathrm{CBC}$ and potentially different toxicity profiles on hematopoiesis impaired by AML involvement.

These different endpoints raise an issue with clinical implications. In this work, Quizartinib was equally effective as Gilteritinib as a single agent in AML carrying both FLT3-ITD and FLT3 wild-type clones. Both FLT3-TKI have been developed in the R/R situation and the phase 3 outcomes showed that Gilteritinib was better for complete remission but just slightly better for overall survival. In a front-line situation, FLT3ITD AML also carries FLT3 wild-type clones and is treated with intensive chemotherapy, which by itself induces deep toxicity in normal hematopoiesis. Quizartinib could be an effective treatment in this case regardless of its action on normal hematopoiesis. Moreover, priming with Quizartinib has recently been shown to inhibit chemotherapy-induced myelosuppression by inducing a transient quiescence of multipotent progenitors, protecting these cells from chemotherapy (32). In contrast, Gilteritinib as single agent is more effective and less toxic than standard chemotherapy, establishing a new standard of care for management of R/R FLT3-mutated AML. Our results suggest that its intrinsic characteristics make it most effective as single agent in high allelic ratio FLT3-ITD AML.

As single agents, FLT3 TKI such as Gilteritinib or others are unlikely to result in a high cure rate for FLT3-ITD AML. Gilteritinib, Quizartinib, or Crenolanib could improve the cure rates for patients with FLT3- 
441 mutated AML, but only when incorporated into a treatment regimen that includes other chemotherapies or 442 drugs. In that setting, ability of Gilteritinib to inhibit AXL could be a leverage to eradicate FLT3-mutated 443 AML clones but could also help to target FLT3 wild-type AML clones shielded by their microenvironment 444 since AXL resistance to chemotherapy is encountered beyond FLT3-ITD AML cells. These data strongly 445 support current clinical trials associating chemotherapy and Gilteritinib.

446 
Figure 1: Activity of gilteritinib against FLT3-ITD AML cell lines

451 A. Relative proliferation of MV4-11, MOLM-13, and MOLM-14 FLT3-ITD AML cell lines incubated in the 452 absence (Vehicle) or with increasing concentration of gilteritinib (as indicated) for 4 days using CellTiter 96 453 AQueous. Condition Vehicle with DMSO $0.1 \%$. The proliferation of the cells treated with the vehicle control 454 was set as $100 \%$ (mean \pm standard error of the mean; $n=4$ ).

B. FLT3-ITD AML cell lines were treated in the absence (Vehicle) or with increasing dose of gilteritinib (as 457 indicated) for $48 \mathrm{~h}$. Apoptosis was measured by Annexin V/DAPI staining followed by flow cytometric 458 analysis. Results are presented as the percentage of the Annexin V cells. Condition Vehicle with DMSO $4590.1 \%$. Mean \pm standard error of the mean; $\mathrm{n}=3$ with ${ }^{*}, \mathrm{p}<0.05 ; * *, \mathrm{p}<0.01 ; * * *, \mathrm{p}<0.001 ; \mathrm{NS}$, 460 nonsignificant.

462 C. Western blot analysis using indicated antibodies performed on lysates from MV4-11, MOLM-13, and 463 MOLM-14 cell lines. Cells were exposed to gilteritinib at the indicated concentrations for $4 \mathrm{~h}$. Condition 0 $464 \mathrm{nM}$ (Vehicle) with DMSO 0.1\%. Results shown are representative of three independent experiments with 465 densitometry. 

condition

A. Apoptosis assessment of FLT3-ITD AML cell lines MV4-11, MOLM-13 and MOLM-14 treated with quizartinib ( $3 \mathrm{nM}$ ) or gilteritinib (100 nM) for $48 \mathrm{~h}$, and cultured without (no stroma, left panel) or with the MS5 stromal cells (stroma, right panel), at $21 \%$ (solid bars) or $1 \%$ (hatched bars) of $\mathrm{O}_{2}$ concentration, using flow cytometry. Condition Vehicle with DMSO $0.1 \%$. Mean \pm standard error of the mean; $n=3$ with $*, p<$ $0.05 ; * *, p<0.01 ; * * *, p<0.001 ; N S$, nonsignificant.

B. MOLM-14 cells were incubated with quizartinib ( $3 \mathrm{nM}$ ) or gilteritinib ( 20 or $100 \mathrm{nM}$ ) for $4 \mathrm{~h}$ and cultivated without (no stroma) or with MS5 stromal cells (stroma) at $21 \%$ or $1 \%$ of $\mathrm{O}_{2}$ concentration, as indicated. Total cell lysates of the samples were subjected to western blot analysis with the indicated antibodies. Results shown are representative of two independent experiments. Condition Vehicle with DMSO $0.1 \%$. 480 Results shown are representative of three independent experiments with densitometry : phosphorylation 481 of protein expression was normalized using total protein expression by calculating the ratio of the 482 phosphorylated protein level to their total protein level counterparts. The ratio of the control condition was 483 normalized to 1Mean of $\mathrm{n}=3$ WB with $*, \mathrm{p}<0.05 ; * *, \mathrm{p}<0.01 ; * * *, \mathrm{p}<0.001$; NS, nonsignificant.

485 C. Primary blasts from 6 FLT3-ITD AML patients cultured with the MS5 stromal cells at $20 \% \mathrm{O}_{2}$, were treated without (vehicle) or with Quizartinib ( $3 \mathrm{nM}$ ) or Gilteritinib (20 or $100 \mathrm{nM}$ ) for 48 hrs. Cells were then stained with Annexin $V$ before cytometer analysis. Condition Vehicle with DMSO $0.1 \%$. Graph shows the mean \pm SEM of results with *, $p<0.05 ;{ }^{* *}, \mathrm{p}<0.01 ; \mathrm{NS}$, non-significant. 
490 Figure 3: Dual AXL/FLT3 inhibition by gilteritinib exerts in vivo a higher pro-apoptotic effect than 491 quizartinib in bone marrow microenvironment

493 A. Dose-dependent inhibition of MV4-11 tumor growth by FLT3-TKI. MV4-11 cells were subcutaneously 494 injected into the limbs of NSG mice at day 0 and treated or not with quizartinib $(5 \mathrm{mg} / \mathrm{kg} / \mathrm{day})$ or at various 495 dosing regimens of gilteritinib $(5,10$, and $30 \mathrm{mg} / \mathrm{kg} /$ day). Tumor volume was measured at the indicated 496 days (mean \pm standard error of the mean; $n=40$ ) with *, $p<0.05 ;{ }^{* *}, p<0.01 ; \mathrm{NS}$, non-significant.

B. Time to relapse after gilteritinib or quizartinib discontinuation. Tumor growth was monitored at the 499 indicated days (mean \pm standard error of the mean; $n=40$ ).

501 C. MV4-11 Luc cells were injected at day $0\left(10^{6}\right.$ cells/mouse $)$ in the retro-orbital sinus vein of busulfan502 pretreated female mice. Mice were then treated or not with quizartinib $(5 \mathrm{mg} / \mathrm{kg} / \mathrm{day}$ ) or gilteritinib (30 $503 \mathrm{mg} / \mathrm{kg} /$ day) from day 7 to day 20 . At day 14 and day 20, animals were analyzed using bioluminescent 504 imaging $(n=30)$. Statistical analyses were performed using the log-rank test. ${ }^{*}, p<0.05 ; * *, p<0.01 ; * * *, p$ $505<0.001 ;$ NS, non-significant. 
509 A. Patient-derived xenografts (PDX) were generated by retro-orbital injections of $10^{6}$ viable primary AML 510 cells in NSG mice previously conditioned. When levels reached at least $10 \%$ human AML cells $\left(\mathrm{CD} 33^{+} \mathrm{CD} 45^{+}\right)$,

511 the mice were sacrificed and bone marrow cells were collected, pooled, and cryopreserved. Two batches of 512 AML PDX cells were generated. The engraftment in each mouse was assessed every 10 days by intra513 femoral puncture analyzed by flow cytometry to measure hCD45 chimerism. The mice were sacrificed when 514 the engraftment was higher than $10 \%$. LOH: loss of heterozygosity.

B. AML PDX cells were injected by retro-orbital route in nonconditioned 8-week-old female NSG mice $\left(10^{6}\right.$ viable PDX cells/mouse). After AML PDX cells engraftment ( $>1 \%$ of human cells in bone marrow; $6-7$ weeks post-transplantation), the mice were divided in three homogeneous groups and treated with vehicle (water $10 \%$ DMSO) or Quizartinib (5 mg/kg/day) or Gilteritinib (30 mg/kg/day) by daily oral gavage during 2 weeks. Three days after the last gavage, the mice were sacrificed, analyzed, and $10^{6} \mathrm{AML}$ PDX cells were serially transplanted into secondary nonconditioned recipients. The secondary recipients were sacrificed and analyzed 9-10 weeks post-transplantation.

C. PDX model from a FLT3-ITD AML patient was used to test the in vivo efficacy of treatment with 525 gilteritinib and quizartinib. The percentage of human $C D 45^{+} / \mathrm{CD} 33^{+}$blast cells in the bone marrow from weeks 8-9 post-transplantation (sacrifice) was determined based on flow cytometric analysis in AML PDX from batch 2 (LOH) at week 7 (before treatment) and week 9 post-transplantation (after treatment). Mean \pm standard error of the mean with *, $p<0.05 ; * *, p<0.01 ; N S$, nonsignificant $(n=10)$.

D. PDX model from a FLT3-ITD AML patient was used to test the in vivo efficacy of treatment with 531 gilteritinib and quizartinib. The percentage of human $\mathrm{CD} 45^{+} / \mathrm{CD} 33^{+}$blast cells in the bone marrow from 532 weeks 8-9 post-transplantation (sacrifice) was determined by flow cytometric analysis in AML PDX from 
533 batch 1 (ratio ITD/wt 0.44 ) at week 7 (before treatment) and week 9 post-transplantation (after treatment).

534 Mean \pm standard error of the mean with *, $p<0.05 ;{ }^{* *}, p<0.01 ; N S$, nonsignificant $(n=5)$.

535 
538 Determination of Murine $\mathrm{CD}^{4} 5^{+}$cells (A), murine Lin ${ }^{+}$cells (B), murine Lin ${ }^{-}$cells (C), and Lin ${ }^{+} \mathrm{c}-\mathrm{Kit}^{\mathrm{High}}$ cells (D)

539 in the bone marrow of NSG mice transplanted or not with AML-PDX cells 3 days post-treatment with 540 vehicle, Quizartinib or Gilteritinib. (E) Murine $\mathrm{CD}^{4} 5^{+}$cells in bone marrow of NSG mice transplanted with 541 AML-PDX with LOH or with ITD/wt at 0.44, 3 days post-treatment with vehicle, Quizartinib or Gilteritinib. (F) 542 Colony forming unit (CFU) assay performed with $10^{4}$ BM cells from non-transplanted NSG mice 3 days post543 treatment with vehicle, Quizartinib or Gilteritinib. Murine Lin- cells (G), murine Lin- Sca- $1^{+}$c-Kit $^{\text {High }}$ cells $(H)$, 544 and $\mathrm{HSC}\left(\mathrm{Lin}^{-} \mathrm{C}-\mathrm{Kit}^{+} \mathrm{SCa}-1^{+} \mathrm{CD} 48^{-} \mathrm{CD} 150^{+}\right)(\mathrm{I})$ in the bone marrow of $\mathrm{C} 57 \mathrm{BI} / 6 \mathrm{~J}$ mice 3 days post-treatment 545 with vehicle, Quizartinib or Gilteritinib. (J) Colony forming unit (CFU) assay performed with $10^{4}$ BM cells 546 from C57BI/6J mice 3 days post-treatment with vehicle, Quizartinib or Gilteritinib. CFU-megakaryocyte 547 (CFU-Mk), CFU-erythroid (CFU-E), Burst Forming Unit (BFU-E), CFU-granulocyte (CFU-G), CFU548 monocyte/macrophage (CFU-M), CFU-granulocyte and monocyte/macrophage (CFU-GM), CFU with all 4 549 lineages granulocyte, erythroid, monocyte/macrophage and megakaryocyte (CFU-GEMM). The data are as 550 mean \pm standard error of the mean $(n=5$ mice per group for non-transplanted mice and $n=15$ for AML551 PDX transplanted mice) with ${ }^{*}, \mathrm{p}<0.05 ;{ }^{* *}, \mathrm{p}<0.01 ;{ }^{* *}, \mathrm{p}<0.001 ; \mathrm{NS}$, non-significant. 
553 Figure 6: Antileukemic effects of gilteritinib and quizartinib in a secondary recipient of FLT3-ITD AML

554 patient-derived xenograft

555

$55610^{6}$ living human CD45+ cells issued from primary recipients were injected into non-conditioned secondary

557 recipients. Percentage of human $\mathrm{CD} 45+/ \mathrm{CD} 33+$ blast cells in the bone marrow 10 weeks post-

558 transplantation, which was determined based on flow cytometric analysis for AML PDX from batch 2 (A)

559 and for AML PDX from batch 1 (B). Mean of five mice per cohort \pm standard error of the mean with *, p<

$560 \quad 0.05 ;{ }^{* *}, \mathrm{p}<0.01 ; * * *, \mathrm{p}<0.001 ; \mathrm{NS}$, nonsignificant.

561 


\section{References}

564 1. Gilliland DG, Griffin JD. The roles of FLT3 in hematopoiesis and leukemia. Blood. 2002;100:1532-42.

565 2. Choudhary C, Brandts C, Schwable J, Tickenbrock L, Sargin B, Ueker A, et al. Activation mechanisms of STAT5 by oncogenic Flt3-ITD. Blood. 2007;110:370-4.

567 3. Schlenk RF, Döhner K, Krauter J, Fröhling S, Corbacioglu A, Bullinger L, et al. Mutations and treatment outcome in cytogenetically normal acute myeloid leukemia. N Engl J Med. 2008;358:1909-18.

4. Smith CC, Wang Q, Chin C-S, Salerno S, Damon LE, Levis MJ, et al. Validation of ITD mutations in FLT3 as a therapeutic target in human acute myeloid leukaemia. Nature. 2012;485:260-3.

5. Stone RM, Mandrekar SJ, Sanford BL, Laumann K, Geyer S, Bloomfield CD, et al. Midostaurin plus Chemotherapy for Acute Myeloid Leukemia with a FLT3 Mutation. N Engl J Med. 2017;

6. Röllig C, Serve H, Hüttmann A, Noppeney R, Müller-Tidow C, Krug U, et al. Addition of sorafenib versus placebo to standard therapy in patients aged 60 years or younger with newly diagnosed acute myeloid leukaemia (SORAML): a multicentre, phase 2, randomised controlled trial. Lancet Oncol. 2015;16:1691-9.

7. Cortes JE, Khaled S, Martinelli G, Perl AE, Ganguly S, Russell N, et al. Quizartinib versus salvage chemotherapy in relapsed or refractory FLT3-ITD acute myeloid leukaemia (QuANTUM-R): a multicentre, randomised, controlled, open-label, phase 3 trial. Lancet Oncol. 2019;20:984-97.

8. Perl AE, Martinelli G, Cortes JE, Neubauer A, Berman E, Paolini S, et al. Gilteritinib or Chemotherapy

9. Sexauer A, Perl A, Yang X, Borowitz M, Gocke C, Rajkhowa T, et al. Terminal myeloid differentiation in vivo is induced by FLT3 inhibition in FLT3/ITD AML. Blood. 2012;120:4205-14.

10. McMahon CM, Canaani J, Rea B, Sargent RL, Qualtieri JN, Watt CD, et al. Gilteritinib induces

11. Graham DK, DeRyckere D, Davies KD, Earp HS. The TAM family: phosphatidylserine sensing receptor tyrosine kinases gone awry in cancer. Nat Rev Cancer. 2014;14:769-85. 
12. Ben-Batalla I, Schultze A, Wroblewski M, Erdmann R, Heuser M, Waizenegger JS, et al. Axl, a prognostic and therapeutic target in acute myeloid leukemia mediates paracrine crosstalk of leukemia cells with bone marrow stroma. Blood. 2013;122:2443-52.

13. Park I-K, Mundy-Bosse B, Whitman SP, Zhang X, Warner SL, Bearss DJ, et al. Receptor tyrosine kinase Axl is required for resistance of leukemic cells to FLT3-targeted therapy in acute myeloid leukemia. Leukemia. 2015;29:2382-9.

14. Dumas P-Y, Naudin C, Martin-Lannerée S, Izac B, Casetti L, Mansier O, et al. Hematopoietic niche drives FLT3-ITD acute myeloid leukemia resistance to quizartinib via STAT5- and hypoxia- dependent

15. Nakao M, Yokota S, Iwai T, Kaneko H, Horiike S, Kashima K, et al. Internal tandem duplication of the up-regulation of AXL. Haematologica. 2019; flt3 gene found in acute myeloid leukemia. Leukemia. 1996;10:1911-8.

16. Guitart AV, Panagopoulou TI, Villacreces A, Vukovic M, Sepulveda C, Allen L, et al. Fumarate hydratase is a critical metabolic regulator of hematopoietic stem cell functions. J Exp Med. 2017;214:719-35.

17. Nair AB, Jacob S. A simple practice guide for dose conversion between animals and human. J Basic Clin Pharm. 2016;7:27-31.

18. Retmana IA, Wang J, Schinkel AH, Schellens JHM, Beijnen JH, Sparidans RW. Liquid chromatographytandem mass spectrometric assay for the quantitative determination of the tyrosine kinase inhibitor quizartinib in mouse plasma using salting-out liquid-liquid extraction. J Chromatogr B Analyt Technol Biomed Life Sci. 2017;1061-1062:300-5.

19. Zarrinkar PP, Gunawardane RN, Cramer MD, Gardner MF, Brigham D, Belli B, et al. AC220 is a uniquely potent and selective inhibitor of FLT3 for the treatment of acute myeloid leukemia (AML). Blood. 2009;114:2984-92.

20. Sandmaier BM, Khaled S, Oran B, Gammon G, Trone D, Frankfurt O. Results of a phase 1 study of quizartinib as maintenance therapy in subjects with acute myeloid leukemia in remission following allogeneic hematopoietic stem cell transplant. Am J Hematol. 2018;93:222-31. 
21. Mori M, Kaneko N, Ueno Y, Yamada M, Tanaka R, Saito R, et al. Gilteritinib, a FLT3/AXL inhibitor, shows antileukemic activity in mouse models of FLT3 mutated acute myeloid leukemia. Invest New Drugs. 2017;

22. James AJ, Smith CC, Litzow M, Perl AE, Altman JK, Shepard D, et al. Pharmacokinetic Profile of Gilteritinib: A Novel FLT-3 Tyrosine Kinase Inhibitor. Clin Pharmacokinet. 2020;

23. Verma A, Warner SL, Vankayalapati H, Bearss DJ, Sharma S. Targeting Axl and Mer kinases in cancer. Mol Cancer Ther. 2011;10:1763-73.

24. Gioia R, Trégoat C, Dumas P-Y, Lagarde V, Prouzet-Mauléon V, Desplat V, et al. CBL controls a tyrosine kinase network involving AXL, SYK and LYN in nilotinib-resistant chronic myeloid leukaemia. J Pathol. 2015;237:14-24.

25. Rochlitz C, Lohri A, Bacchi M, Schmidt M, Nagel S, Fopp M, et al. Axl expression is associated with adverse prognosis and with expression of $\mathrm{Bcl}-2$ and $\mathrm{CD} 34$ in de novo acute myeloid leukemia (AML): results from a multicenter trial of the Swiss Group for Clinical Cancer Research (SAKK). Leukemia. 1999;13:1352-8.

26. Whitman SP, Kohlschmidt J, Maharry K, Volinia S, Mrózek K, Nicolet D, et al. GAS6 expression identifies high-risk adult AML patients: potential implications for therapy. Leukemia. 2014;28:1252-8.

27. Hong C-C, Lay J-D, Huang J-S, Cheng A-L, Tang J-L, Lin $M-T$, et al. Receptor tyrosine kinase AXL is induced by chemotherapy drugs and overexpression of AXL confers drug resistance in acute myeloid leukemia. Cancer Lett. 2008;268:314-24.

28. Park I-K, Mishra A, Chandler J, Whitman SP, Marcucci G, Caligiuri MA. Inhibition of the receptor tyrosine kinase Axl impedes activation of the FLT3 internal tandem duplication in human acute myeloid leukemia: implications for Axl as a potential therapeutic target. Blood. 2013;121:2064-73.

29. Korn C, Méndez-Ferrer S. Myeloid malignancies and the microenvironment. Blood. 2017;129:811-22.

30. Perl AE, Altman JK, Cortes J, Smith C, Litzow M, Baer MR, et al. Selective inhibition of FLT3 by gilteritinib in relapsed or refractory acute myeloid leukaemia: a multicentre, first-in-human, openlabel, phase 1-2 study. Lancet Oncol. 2017; 
640 31. Cortes J, Perl AE, Döhner H, Kantarjian H, Martinelli G, Kovacsovics T, et al. Quizartinib, an FLT3 641 inhibitor, as monotherapy in patients with relapsed or refractory acute myeloid leukaemia: an open642 label, multicentre, single-arm, phase 2 trial. Lancet Oncol. 2018;

643 32. Taylor SJ, Duyvestyn JM, Dagger SA, Dishington EJ, Rinaldi CA, Dovey OM, et al. Preventing 644 chemotherapy-induced myelosuppression by repurposing the FLT3 inhibitor quizartinib. Sci Transl $645 \quad$ Med. 2017;9.

646 


\section{Acknowledgements}

648 We thank Mrs. Bernadette De Buhan and her family for their generous support. This work was supported 649 by grants from Ligue contre le cancer (Comité des Pyrénées-Atlantiques). We thank the Cytometry Facility, 650 Vectorology Facility, Cell'Oxia and Animals Facility A2 of the FRTransBioMed, Bordeaux University. P.-Y.D. 651 was a recipient of the SIRIC Brio for research fellowship for one year, then the MD-PhD program from the 652 University Hospital of Bordeaux for two years. We also acknowledge the Centre de Ressources Biologiques 653 Cancer, Bordeaux Biothèques Santé (BB-0033-00036) at Bordeaux University Hospital for providing 654 biological material.

\section{Authorship}

657 Designed research and analyzed data, P.-Y.D., A.V., J.-M.P. and V.D.; Performed experiments, P.-Y.D., A.V., 658 A.G., L.M., D.M., S.F. and V.D.; Animal experiments, P.-Y.D., J.-M.P., A.V. and A.G.; Primary AML sample, 659 conditioning, delivery, P.-Y.D., A.P., T.L., A.B., O.M.; Comments on the research direction and edition of the 660 manuscript A.P. and I.V.; Original Draft, Writing \& Edition, P.-Y.D. and V.D.; Made Figures, P.-Y.D. and V.D.; 661 Funding, P.-Y.D. and V.D.

662

663 We thank Prof. C. Récher for his critical reading of the manuscript.

665 The English in this document has been checked by at least two professional editors, both native speakers of 666 English. For a certificate, please see:

667 http://www.textcheck.com/certificate/AnQDrr 668 
670 Pierre-Yves Dumas: Advisory boards: Daiichi-Sankyo, Astellas

671 Arnaud Villacreces: no disclosure

672 Amelie Guitart: no disclosure

673 Layal Massara: no disclosure

674 Olivier Mansier: no disclosure

675 Audrey Bidet: Advisory board: Daiichi-Sankyo, Novartis

676 Delphine Martineau: no disclosure

677 Solene Fernandez: no disclosure

678 Thibaut Leguay: no disclosure

679 Arnaud Pigneux: Research grants (my institution): Incyte, Janssen, Gilead, Sanofi, Amgen, Novartis, Celgene,

680 Jazz Pharma, Daiichi-Sankyo, Astellas, Roche. Advisory boards: Sanofi, Takeda, Abbvie, Janssen, Jazz

681 Pharma, Daiichi-Sankyo, Astellas, Novartis, Celgene, Otsuka, Sunesis, Roche, Pfizer.

682 Isabelle Vigon: no disclosure

683 Jean-Max Pasquet: no disclosure

684 Vanessa Desplat: no disclosure

685

686

687

688

689

690

691

692

693

694 
696 